\title{
USO DEL ELECTROENCEFALOGRAMA INTRAOPERATORIO: EVALUACIÓN PRELIMINAR DE LOS CAMBIOS EN LA POTENCIA RELATIVA DEL PATRÓN THETA DURANTE UNA CIRUGÍA ABDOMINAL MAYOR
}

Gutiérrez Rojas Rodrigo $^{1}$, Egaña Tomic José Ignacioํㅜ ${ }^{1}$ Penna Silva Antonello ${ }^{1}$

1 Departamento de Anestesiología y Reanimación, Universidad de Chile.

Introducción: Recientemente ha habido un creciente interés en el uso del electroencefalograma como parte del monitoreo cerebral intraoperatorio, sobre todo mediante el análisis del espectrograma. Este consiste en estudiar la señal electroencefalográfica en el dominio tiempofrecuencia, lo que permite cuantificar la potencia relativa de una determinada frecuencia en cierto momento.

Objetivo General: Determinar la potencia relativa de la frecuencia theta intraoperatoria, en pacientes sometidos a cirugía abdominal mayor con anestesia general, mediante el análisis del espectrograma.

Material y Métodos: Se llevó a cabo un estudio prospectivo, longitudinal, observacional y analítico en el HCUCH. Se reclutaron pacientes mayores de 60 años con consentimiento firmado. Se excluyeron pacientes ASA III-V, con antecedentes de enfermedad neurológica, deterioro cognitivo de origen médico, enfermedad psiquiátrica mayor y pacientes con delirium previo a la cirugía. Tras aceptar participar del estudio se instaló a cada paciente un EEG de 16 canales
15 minutos previo al inicio de la cirugía. Luego se realizó un registro de aproximadamente $5 \mathrm{mi}-$ nutos previo a la inducción, y que corresponde al basal. Durante la cirugía se registra de forma continua. Además se registraron otras variables de interés como la concentración alveolar mínima, variables hemodinámicas, entre otras. El procesamiento de la señal y su análisis fue realizado utilizando Matlab.

Resultados: A la fecha se han reclutado 7 pacientes. En el presente resumen se describe de forma preeliminar el cambio en la potencia relativa de theta previo a la cirugía y luego durante esta, en un paciente sometido a una gastrectomía total. Previo a la inducción anestésica este paciente presentó una potencia relativa de theta de un $10 \%$. Una vez que se induce la anestesia general con un bolo de propofol $(2 \mathrm{mg} / \mathrm{kg})$ la potencia relativa theta aumenta a $22 \%$. Luego, en el intraoperatorio se determinó la potencia relativa de theta cada 30 minutos, obteniendose las siguientes mediciones: $23 \%, 22 \%, 17 \%, 12 \%$, $14 \%$ y $13 \%$. Para cada una de estas mediciones de la potencia relativa de theta se registró el valor

Tabla 1

\begin{tabular}{|c|c|c|c|c|c|}
\hline & Tiempo (segs) & $\begin{array}{l}\text { Potencia Theta } \\
\text { (uV2/hz) }\end{array}$ & $\begin{array}{l}\text { Potencia } \\
\text { (uV2/hz) }\end{array}$ & $\begin{array}{l}\text { Relación Theta/ } \\
\text { Total }\end{array}$ & $\begin{array}{l}\text { End tidal } \\
\text { Sevofluorano }\end{array}$ \\
\hline Despierto & & 45,42 & 431,63 & 0,105229016 & 0 \\
\hline \multirow[t]{7}{*}{ Dormido } & $0-120$ & 97,2 & 431,94 & 0,225031254 & 1,2 \\
\hline & $1.800-2.000$ & 104,06 & 451,45 & 0,230501717 & 1,6 \\
\hline & $3.600-3.800$ & 158,62 & 704,21 & 0,22524531 & 1,5 \\
\hline & $5.400-5.600$ & 89,66 & 510,75 & 0,175545766 & 1,5 \\
\hline & $7.200-7.400$ & 81,85 & 638,61 & 0,128168992 & 1,8 \\
\hline & $9.000-9.200$ & 89,03 & 600,28 & 0,14831412 & 1,9 \\
\hline & 10.000 & 61,61 & 444,72 & 0,138536607 & 1,2 \\
\hline
\end{tabular}


de la concentración de sevoflorano en aire espirado, obteniéndose los siguientes resultados: 1.2 , $1.6,1.5,1.5,1.8,1.9$, y 1.2 respectivamente. Estos resultados se resumen en la tabla.

Conclusiones: La realización de un monitoreo electroencefalográfico es factible en el ambiente perioperatorio. La potencia relativa del patron theta no es constante durante una cirugía. El principal cambio ocurre al momento de la inducción, tras la cual aumenta la potencia relativa de theta. De forma preliminar se observa que a mayor concentración de sevoflorano en aire espi- rado, menor es la potencia relativa de theta en el espectrograma.

\section{Referencias}

1. Purdon PL, Sampson A, Pavone KJ, Brown EN. Clinical Electroencephalography for Anesthesiologists: Part I: Background and Basic Signatures. Anesthesiology 2015; 123:937-60.

Financiamiento: Proyecto FONDECYT de Iniciación. 